\title{
Asymptotic behaviour of large solutions of quasilinear elliptic problems
}

Catherine Bandle

To L. E. Payne on the occasion of his 80th anniversary

Abstract. The paper deals with the large solutions of the problems $\triangle u=u^{p}$ and $\triangle u=e^{u}$. They blow up at the boundary. It is well-known that the first term in their asymptotic behaviour near the boundary is independent of the geometry of the boundary. We determine the second term which depends on the mean curvature of the nearest point on the boundary. The computation is based on suitable upper and lower solutions and on estimates given in [4]. In the last section these estimates are used together with the $P$-function to establish the asymptotic behaviour of the gradients

Mathematics Subject Classification (2000). 35B45, 35J25, 35J60.

Keywords. Nonlinear boundary value problems, upper and lower solutions, boundary blowup, maximal solutions.

\section{Introduction}

In this paper we consider problems of the type

$$
\triangle u=f(u) \text { in } D, u(x) \rightarrow \infty \text { as } x \rightarrow \partial D,
$$

where $f: \mathbb{R} \rightarrow \mathbb{R}^{+}$is a nondecreasing function. The solutions of (1.1) are called large solutions. If $\partial D$ is bounded and satisfies an inner and outer sphere condition J. Keller [10] has shown that such solutions exist if and only if

$$
\int^{\infty} \frac{d t}{\sqrt{2 F(t)}} d t<\infty, \text { where } F^{\prime}(t)=f(t) .
$$

Typical examples are $f(t)=t^{p}$ with $p>1$ and $f(t)=e^{t}$. In these cases Problem (1.1) has a unique solution.

The asymptotic behaviour of the large solutions near the boundary has been studied in a series of papers [1]-[6], [8], [9]. It turns out that the first order 
approximation depends only on the distance $\delta(x)=\operatorname{dist}\{x, \partial D\}$ and not on the geometry of the domain $D$. The expressions for the asymptotic behaviour are particularly simple for power nonlinearities $f(t)=t^{p}$, namely

$$
u(x)=(\gamma \delta)^{-\frac{2}{p-1}}(1+o(1)) \text { as } x \rightarrow \partial D, \gamma=\frac{p-1}{\sqrt{2(p+1)}}
$$

and for the exponential function $f(t)=e^{t}$ where

$$
u(x)=\log \frac{2}{\delta^{2}}(1+o(1)) \text { as } x \rightarrow \partial D .
$$

The problem of the second order effects was first discussed by Lazer and McKenna [9]. They proved that for $f(t)=t^{p}$ with $p>3$ and $f(t)=e^{t}$

$$
\lim _{x \rightarrow \partial D}\left(u(x)-(\gamma \delta)^{-\frac{2}{p-1}}\right)=0, \text { and } \lim _{x \rightarrow \partial D}\left(u(x)-\log \frac{2}{\delta^{2}}\right)=0 .
$$

The main results of this paper are the asymptotic expansions given in

Theorem 1. Let $\partial D \in C^{4}$ be compact and let $\bar{x}$ be the nearest point to $x$ on $\partial D$. Let $H(\bar{x})$ denote the mean curvature of $\partial D$ at $\bar{x}$. Then for $f(t)=t^{p}$, $p>1$ we have

$$
u(x)=(\gamma \delta)^{-\frac{2}{p-1}}\left(1+\frac{(N-1) H(\bar{x})}{p+3} \delta+o(\delta)\right) \text { as } x \rightarrow \partial D .
$$

For $f(t)=e^{t}$ we have

$$
u(x)=\log \frac{2}{\delta^{2}}+(N-1) H(\bar{x}) \delta+o(\delta) \text { as } x \rightarrow \partial D
$$

(1.2) and (1.3) have been established in [5] for balls and annuli. Del Pino and Letelier [6] have extended (1.2) to general domains in the case where $p<3$. The proof of Theorem 1 relies on suitable upper and lower solutions which have been constructed [6], and on estimates derived in [5]. A general approach to these types of expansions is discussed in [1].

Finally we use Theorem 1 together with gradient estimates of [2] and [3] to establish the asymptotic behaviour of the gradients near the boundary. These estimates are based on the $P$-function which has been studied in a number of papers by L.E. Payne. He has first discussed it in [11] in connection with the torsion problem and generalized it later on to quasilinear elliptic and parabolic problems. For further references see [12]. The method presented here can be used to discuss the second term in the asymptotic behaviour for problems with more general nonlinearities. This extension will be the carried out in a forthcoming paper. 


\section{Proof of Theorem 1}

Let as before $\bar{x}$ be the projection of the point $x \in D$ on $\partial D, \rho$ measures the distance of $x$ to the boundary. It is well-known (cf. e.g. [7], p. 382) that if $\partial D \in C^{k}, k \geq 2$, then there exists $\mu>0$ such that $\delta(x) \in C^{k}\left(D_{\mu}\right)$ where $D_{\mu}=\{x \in D: \delta(x)<\mu\}$. Moreover for $\mu$ sufficiently small $(\bar{x}, \rho)$ can be taken as new coordinates in $D_{\mu}$. In these coordinates the Laplacian becomes

$$
\triangle_{x}=\triangle_{\partial D_{\rho}}+\frac{\partial^{2}}{\partial \rho^{2}}-(N-1) H(x) \frac{\partial}{\partial \rho},
$$

where $\triangle_{\partial D_{\rho}}$ is the Laplace-Beltrami operator on $\partial D_{\rho}$ and $H(x)$ is the mean curvature of $\partial D_{\rho}$ at $x$. It should be observed that $\triangle_{\partial D_{\rho}}$ and $H(x)$ can be computed from the differential geometric quantities of $\partial D$. If $\kappa_{i}(\bar{x}), i=1, \ldots, N-1$ are the principal curvatures of $\partial D$ at $\bar{x}$, then (c.f. [7]),

$$
H(x)=\frac{1}{N-1} \sum_{i=1}^{N-1} \frac{\kappa_{i}}{1-\rho \kappa_{i}} .
$$

For a domain in the plane we have

$$
\triangle_{x}=(1-\kappa \rho)^{-1} \frac{\partial}{\partial s}\left((1-\kappa \rho)^{-1} \frac{\partial}{\partial s}\right)+\frac{\partial^{2}}{\partial \rho^{2}}-\frac{\kappa}{1-\kappa \rho} \frac{\partial}{\partial \rho} .
$$

Here $s$ is the arc length of $\partial D$ and $\kappa$ is the curvature of $\partial D$ at the point $s$. From these considerations it follows that

$$
\triangle \delta(x)=-(N-1) H(x)=-(N-1) H(\bar{x})+O(\delta) \text { as } \delta \rightarrow 0 .
$$

For small positive $\epsilon$ and $\nu$ set

$$
v_{ \pm}(x)=\left(\gamma \delta_{\mp \epsilon}(x)\right)^{-\frac{2}{p-1}}\left(1+\frac{(N-1) H_{ \pm \nu}}{p+3} \delta_{\mp \epsilon}(x)\right),
$$

where

$$
\delta_{\mp \epsilon}=\delta \mp \epsilon \text { and } H_{ \pm \nu}=H(\bar{x}) \pm \nu .
$$

Similarly we define

$$
w_{ \pm}(x)=\log \frac{2}{\delta_{\mp \epsilon}^{2}(x)}+(N-1) H_{ \pm \nu} \delta_{\mp \epsilon}(x) .
$$

Notice that $v_{ \pm}$and $w_{ \pm}$are the expected first two terms in the asymptotic expansion of the large solutions corresponding to $t^{p}$ and $e^{t}$. If $\partial D \in C^{4}$, the mean 
curvature $H(\bar{x})$ is in $C^{2}$. A straightforward computation yields

$$
\begin{array}{r}
\triangle v_{ \pm}=\left\{1+\frac{2 \gamma^{2}(N-1)}{p-1}\left[H(x)-\frac{H_{ \pm \nu}(p-3)}{(p+3)(p-1)}\right] \delta_{\mp \epsilon}-\right. \\
\left.\frac{(N-1)^{2} H(x) H_{ \pm \nu}(p-3)}{(p+3)(p-1)} \delta_{\mp \epsilon}^{2}+O\left(\delta \delta_{\mp \epsilon}\right)\right\}\left(\gamma \delta_{\mp \epsilon}\right)^{-\frac{2 p}{p-1}}= \\
\left\{1+\frac{p(N-1) H_{ \pm \nu}}{p+3} \delta_{\mp \epsilon} \mp \frac{2 \gamma^{2}(N-1) \nu}{p-1} \delta_{\mp \epsilon}+\eta\right\}(\gamma \delta)^{-\frac{2 p}{p-1}},
\end{array}
$$

where $\eta=\omega \delta_{\mp \epsilon} \delta+O\left(\delta_{\mp \epsilon}^{2}\right)$ and $|\omega|<c$ in $D_{\mu}$. The expression $O\left(\delta \delta_{\mp \epsilon}\right)$ contains tangential derivatives of $H(\bar{x})$ with respect to $\partial D$. Similarly

$$
\begin{gathered}
\triangle w_{ \pm}=\frac{2}{\delta_{\mp \epsilon}^{2}}\left\{1+(N-1) H(x) \delta_{\mp \epsilon}-\frac{N-1}{2} H(x) H_{ \pm \nu} \delta_{\mp \epsilon}^{2}\right\}+ \\
O\left(\delta_{\mp \epsilon}\right)=\frac{2}{\delta_{\mp \epsilon}^{2}}\left\{1+(N-1) H(\bar{x}) \delta_{\mp \epsilon}+O\left(\delta \delta_{\mp \epsilon}\right)+O\left(\delta_{\mp \epsilon}^{2}\right)\right\} .
\end{gathered}
$$

The next result is due to del Pino and Letelier [6].

Lemma 1. There exists a positive continuous function $\nu(\mu)$ with $\nu(\mu) \rightarrow 0$ as $\mu \rightarrow 0$ such that

$$
\triangle v_{+} \leq v_{+}^{p} \text { and } \triangle w_{+} \leq e^{w_{+}} \text {in } D_{\mu}
$$

Analogously

$$
\triangle v_{-} \geq v_{-}^{p} \text { and } \triangle w_{-} \leq e^{w_{-}} \text {in } D_{\mu}
$$

Proof. From (2.3) we find for small $\delta_{\epsilon}$

$$
\triangle v_{+}=v_{+}^{p}-\left(\frac{2 \gamma^{2}(N-1) \nu}{p-1} \delta_{-\epsilon}+\omega \delta_{-\epsilon} \delta+O\left(\delta_{-\epsilon}^{2}\right)\right)\left(\gamma \delta_{-\epsilon}\right)^{-\frac{2 p}{p-1}} .
$$

It is now obvious that $\nu$ can be chosen in such a way that the paranthesis on the right-hand side becomes negative and that it vanishes as $\delta$ tends to zero. If we replace $\epsilon$ and $\nu$ by $-\epsilon$ and $-\nu$ we obtain the statement concerning $v_{-}$.

In order to prove the inequalities for $w_{\mp}$ we observe that (2.4) assumes the form

$$
\triangle w_{+}=e^{w_{+}}-\frac{2}{\delta_{-\epsilon}^{2}}\left\{(N-1) \nu \delta_{-\epsilon}+O\left(\delta \delta_{-\epsilon}\right)+O\left(\delta_{-\epsilon}^{2}\right)\right\},
$$

and similarly

$$
\triangle w_{-}=e^{w_{-}}+\frac{2}{\delta_{+\epsilon}^{2}}\left\{(N-1) \nu \delta_{+\epsilon}+O\left(\delta \delta_{+\epsilon}\right)+O\left(\delta_{+\epsilon}^{2}\right)\right\},
$$


The remainder of the proof is now obvious.

In the proof of Theorem 1 we shall make use of a result of [5].

Lemma 2. Under the assumptions of Theorem 1 the large solutions corresponding to $f(t)=t^{p}$ and $f(t)=e^{t}$, resp. satisfy in $D_{\mu}$

$$
\left|u(x)-(\gamma \delta)^{-\frac{2}{p-1}}\right| \leq c(\gamma \delta)^{-\frac{2}{p-1}+1} \text { and }\left|u(x)-\log \frac{2}{\delta^{2}}\right| \leq c \delta \log \frac{2}{\delta^{2}}
$$

We are now in position to prove Theorem 1.

Proof of Theorem 1 Consider the functions $v_{+}$where $\nu(\mu)$ is chosen such that Lemma 1 holds. Since $\epsilon$ is positive we have $v_{+} \geq u$ on $\{x \in D: \delta(x)=\epsilon\}$. Let us now determine $\epsilon$ such that $v_{+} \geq u$ on the inner boundary $\partial D_{\mu} \cap D$. In view of Lemma 2 we must have

$$
(\gamma \mu)^{-\frac{2}{p-1}}\left(1-\frac{\epsilon}{\mu}\right)^{-\frac{2}{p-1}}(1+O(\mu)) \geq(\gamma \mu)^{-\frac{2}{p-1}}(1+c \mu) \text { for some fixed } c .
$$

This inequality holds if $1>\mu \geq \epsilon \geq c_{0} \mu^{2}$ for some suitable constant $c_{0}$. By the maximum principle we have $u \leq v_{+}$in $\{x: \epsilon<\delta(x)<\mu\}$.

Similarly we choose $\nu$ such that Lemma 1 holds for $v_{-}$. Obviously $v_{-}<u$ on $\partial D$. As before we can achieve by choosing a suitable $\epsilon$ such that $1>\mu \geq \epsilon \geq$ $c_{1} \mu^{2}$, that $v_{-} \leq u$ on $\partial D_{\mu} \cap D$. From the maximum principle we conclude that $v_{-} \leq u$ in $D_{\mu}$. Put $\mu=2 \epsilon$. (1.2) follows from these comparisons together with the fact that $\nu(\mu) \rightarrow 0$ and $\epsilon(\mu) \rightarrow 0$ as $\mu \rightarrow 0$.

In the same way by choosing $\epsilon \geq c \mu^{2} \log \frac{2}{\delta^{2}}$ we can achieve that $w_{-} \leq u \leq w_{+}$. This completes the proof of the theorem.

\subsection{Extensions and remarks}

The term $o(\delta)$ in (1.2) can be made more precise. As it was observed before $\epsilon$ and $\nu$ can be chosen such that $v_{-} \leq u \leq v_{+}$in a small parallel strip $D_{\mu}$. This is the case if $\nu=c_{0} \mu$ and $\epsilon=c_{1} \mu^{2}$. Hence for small $\delta$

$$
u(x)=(\gamma \delta)^{-\frac{2}{p-1}}\left(1+\frac{(N-1) H(\bar{x})}{p+3} \delta+O(\mu) \delta\right) .
$$

Choosing $\mu=2 \epsilon$ we obtain the following extension of Lazer and McKenna's result mentioned in the Introduction.

Corollary 1. Under the same assumptions as in Theorem 1 we have for any $p>2$

$$
u(x)-(\gamma \delta)^{-\frac{2}{p-1}}\left(1+\frac{(N-1) H(\bar{x})}{p+3} \delta\right) \rightarrow 0 \text { as } \delta \rightarrow 0 .
$$


Notice that in the case where $H(\bar{x})=0$ we have $\lim _{\delta \rightarrow 0}\left[u(x)-(\gamma \delta)^{-\frac{2}{p-1}}\right]=0$ for any $p>2$. Then the solution behaves locally like the large solutions in a strip.

Examples Consider the large solution of $\triangle u=u^{\frac{N+2}{N-2}}$ in $D \subset \mathbb{R}^{N}$. This problem plays an import role in conformal geometry. In the unit ball the solution is known explicitely, namely

$$
u(x)=\{N(N-2)\}^{\frac{N-2}{2}}\left(1-|x|^{2}\right)^{-\frac{N-2}{2}}=\left\{\frac{\sqrt{N(N-2)}}{2 \delta}\right\}^{\frac{N-2}{2}}\left(1-\frac{\delta}{2}\right)^{-\frac{N-2}{2}} .
$$

The statement of the Corollary 1 holds true if $N<6$.

Consider the large solution of $\triangle u=e^{u}$ in a domain $D$ in the plane. The large solution of the unit circle is given by

$$
u(x)=\log \frac{8}{\left(1-|x|^{2}\right)^{2}} .
$$

Observe that the equation is invariant under conformal mappings in the following sense:

Let $z=x_{1}+i x_{2}$ be the complex variable in the plane and $f: D \rightarrow D^{\prime} \subset \mathbb{C}$ be a conformal mapping. If $u(z)$ is a solution in $D^{\prime}$ then $u(f(z))+\log \left|f^{\prime}(z)\right|^{2}$ is a solution in $D$. This simple invariance property together with the explicit solution in the unit circle leads to a class of large solutions already known to Liouville (1856). Indeed if $D$ is simply connected and $f: D \rightarrow\{|z|<1\}$ is a conformal mapping then

$$
u(z)=\log \frac{8\left|f^{\prime}\right|^{2}}{\left(1-|f|^{2}\right)^{2}}
$$

is the large solution in $D$. Notice that it doesn't depend on the special choice of $f$. From Theorem 1 or by direct computation we obtain an expression for the behaviour of $f$ near the boundary

$$
\frac{8\left|f^{\prime}(z)\right|^{2}}{\left(1-|f(z)|^{2}\right)^{2}}=\frac{2}{\delta^{2}}\left(1+\kappa \delta+O\left(\delta^{2}\right)\right)
$$

where $\delta=\operatorname{dist}(z, \partial D)$.

\section{Asymptotic behaviour of the gradients}

It was shown in [2], (cf. also [4]) that the derivatives of the large solutions in the direction of the inner normal satisfy

$$
\begin{array}{r}
\frac{\partial u}{\partial \rho}=-\frac{2}{p-1} \gamma^{-\frac{2}{p-1}} \delta^{-\frac{p+1}{p-1}}(1+o(1)) \text { as } \delta \rightarrow 0, \text { if } f=t^{p} \\
\frac{\partial u}{\partial \rho}=-2 \delta^{-1}(1+o(1)) \text { as } \delta \rightarrow 0, \text { if } f=e^{t}
\end{array}
$$


For domains $D$ of nonnegative mean curvature the P-function [12] leads to the following estimate [3]

$$
\begin{array}{r}
|\nabla u|^{2} \leq \frac{2}{p+1}\left(u^{p+1}-u_{\min }^{p+1}\right) \text { if } f=t^{p}, \\
|\nabla u|^{2} \leq 2\left(e^{u}-e^{u_{\text {min }}}\right) \text { if } f=e^{t} .
\end{array}
$$

Taking into account (1.2) and (1.3) we obtain the following behaviour of $\nabla u$ near the boundary

$$
\begin{array}{r}
|\nabla u| \leq \frac{2}{p-1} \gamma^{-\frac{2}{p-1}} \delta^{-\frac{p+1}{p-1}}\left(1+\frac{p(N-1)}{2(p+3)} H(\bar{x}) \delta+o(\delta)\right) \text { if } f=t^{p}, \\
|\nabla u| \leq \frac{2}{\delta}\left(1+\frac{N-1}{2} H(\bar{x}) \delta\right)+O(\delta) \text { if } f=e^{t} .
\end{array}
$$

The same reasoning as in the Corollary 1 shows that $o(\delta)=O\left(\delta^{2}\right)$. Hence $(3.1)$, (3.3) and (3.2), (3.4) imply

Theorem 2. Under the same assumptions as for Theorem 1 and if in addition $\partial D$ has positive mean curvature then we have for $f=t^{p}$ with $p>3$

$$
|\nabla u|-\frac{2}{p-1} \gamma^{-\frac{2}{p-1}} \delta^{-\frac{p+1}{p-1}}\left(1+\frac{p(N-1)}{2(p+3)} H(\bar{x}) \delta\right) \rightarrow 0 \text { as } \delta \rightarrow 0 .
$$

If $f=e^{t}$ we have

$$
|\nabla u|-\frac{2}{\delta}\left(1+\frac{N-1}{2} H(\bar{x}) \delta\right) \rightarrow 0 \text { as } \delta \rightarrow 0 .
$$

\section{References}

[1] L. Andersson, P. T. Chruściel, Solutions of the constraint equation in general relativity satisfying "hyperbolic conditions", Dissertationes Matematicae CCCLV (1996).

[2] C. Bandle, M. Essèn, On the solutions of quasilinear elliptic problems with boundary blowup, Symposia Matematica 35 (1994), 93-111.

[3] C. Bandle, M. Marcus, Large solutions of semilinear elliptic equations: existence, uniqueness and asymptotic behaviour, J. d'Anal. Mathém. 58 (1992), 9-24.

[4] C. Bandle, M. Marcus, Asymptotic behaviour of solutions and their derivatives, for semilinear elliptic problems with blowup on the boundary, Ann. Inst. Henri Poincaré 12 (1995), $155-171$.

[5] C. Bandle, M. Marcus, On second order effects in the boundary behaviour of large solutions of semilinear elliptic problems, Differential Integral Equ. 11 (1998), 23-34.

[6] M. del Pino, R. Letelier, The influence of domain geometry in boundary blow-up elliptic problems, Nonlinear Anal. TMA 48 (2002), 897-904.

[7] D. Gilbarg, N.S. Trudinger, Elliptic partial differential equations of second order, Springer, Grundlehren der mathematischen Wissenschaften 224 (1977).

[8] A. Greco, G. Porru, Asymptotic estimates and convexity of large solutions to semilinear elliptic equations Differential Integral Equ. 10 (1997), 219-229. 
[9] A. C. Lazer, P. J. McKenna, Asymptotic behaviour of solutions of boundary blow up problems, Differential Integral Equations 7 (1994), 1001-1019.

[10] J. B. Keller, On solutions of $\triangle u=f(u)$, Comm. Pure Appl. Math. 10 (1957), 503-510.

11] L. E. Payne, Bounds for the maximal stress in the St. Venant torsion problem, Indian J. Mech. Math. special issue (1968), 51-59.

12] R. P. Sperb, Maximum principles and their applications, Academic Press, Mathematics in Sciences and Engineering 157 (1981).

Catherine Bandle

Mathematisches Institut

Universität Basel

Rheinsprung 21

CH-4051 Basel

Switzerland

e-mail: bandle@math.unibas.ch

(Received: April 28, 2003) 